\title{
NOTES ON $K$-TOPOLOGICAL GROUPS AND HOMEOMORPHISMS OF TOPOLOGICAL GROUPS
}

\author{
HANFENG WANG and WEI HE ${ }^{凶}$ \\ (Received 15 May 2012; accepted 10 June 2012; first published online 30 August 2012)
}

\begin{abstract}
In this paper, it is shown that there exists a connected topological group which is not homeomorphic to any $\omega$-narrow topological group, and also that there exists a zero-dimensional topological group $G$ with neutral element $e$ such that the subspace $X=G \backslash\{e\}$ is not homeomorphic to any topological group. These two results give negative answers to two open problems in Arhangel'skii and Tkachenko [Topological Groups and Related Structures (Atlantis Press, Amsterdam, 2008)]. We show that if a compact topological group is a $K$-space, then it is metrisable. This result gives an affirmative answer to a question posed by Malykhin and Tironi ['Weakly Fréchet-Urysohn and Pytkeev spaces', Topology Appl. 104 (2000), 181-190] in the category of topological groups. We also prove that a regular $K$-space $X$ is a weakly Fréchet-Urysohn space if and only if $X$ has countable tightness.
\end{abstract}

2010 Mathematics subject classification: primary 54H11; secondary 54E35, $22 \mathrm{~A} 05$.

Keywords and phrases: $\omega$-narrow topological group, zero-dimensional topological group, $K$-space, countable tightness.

\section{Introduction}

This paper consists of two parts. In the first part, we consider two open problems posed by Arhangel'skii and Tkachenko in [2]. The first (Open Problem 3.4.6) asks whether every connected topological group is homeomorphic to an $\omega$-narrow topological group. The second (Open Problem 1.4.1) asks whether for a zero-dimensional topological group $G$ with neutral element $e$, the space $X=G \backslash\{e\}$ is homeomorphic to a topological group. In this paper we will show that there exists a connected topological group which is not homeomorphic to any $\omega$-narrow topological group, and this gives a negative answer to the first open problem. We show that there exists a zero-dimensional topological group $G$ with neutral element $e$ such that the subspace $X=G \backslash\{e\}$ is not homeomorphic to any topological group, and this gives a negative answer to the second open problem.

In the second part, we consider an open problem posed by Malykhin and Tironi in [9] for topological spaces. This asks whether a compact $K$-space $X$ must have countable tightness. We restrict our attention to the category of topological groups,

Project supported by NSFC (11171156).

(C) 2012 Australian Mathematical Publishing Association Inc. 0004-9727/2012 \$16.00 
and prove that a compact topological group which is a $K$-space must be metrisable. This gives an affirmative answer to the open problem in the category of topological groups. Moreover, we prove a stronger result that every locally compact topological group which is a $K$-space must be metrisable.

Recall that a topological group $G$ is a group $G$ with a topology such that the product mapping of $G \times G$ into $G$ is jointly continuous and the inverse mapping of $G$ onto itself associating $x^{-1}$ with arbitrary $x \in G$ is continuous. Obviously, each topological group is homogeneous. Thus, to define a topological group topology on a group $G$, it is enough to define a local base at the identity $e$ of $G$ and then translate it to all points in $G$.

A topological group $G$ is called $\omega$-narrow [5] if and only if for every open neighbourhood $V$ of the neutral element $e$ in $G$, there exists a countable subset $A$ of $G$ such that $A V=G$. The class of $\omega$-narrow topological groups contains all Lindelöf topological groups and all topological groups with countable cellularity. Also, $\omega$-narrow topological groups are characterised as subgroups of topological products of families of second countable topological groups (see [5]).

A topological space $X$ is called a $K$-space [9] if, for every $x \in X$ and $B \subset X$ satisfying $x \in \bar{B} \backslash B$, there exists a sequence $\zeta=\left\{K_{i}: i \in \omega\right\}$ of disjoint compact subsets of $B$ such that, for every neighbourhood $U$ of $x,\left\{i \in \omega: K_{i} \cap U=\emptyset\right\}$ is finite. For convenience, we denote this relation of $x$ and $\zeta$ by $x(K) \zeta$.

We say that the tightness of a topological space $X$ is countable if, for each $x \in X$ and $A \subset X$ satisfying $x \in \bar{A}$, there exists a countable subset $B$ of $A$ such that $x \in \bar{B}$.

Recall that a topological space $X$ is called a weakly Fréchet-Urysohn space [9] if, for each $x \in X$ and $A \subset X$ satisfying $x \in \bar{A} \backslash A$, there exists a sequence $\zeta=\left\{F_{i}: i \in \omega\right\}$ consisting of disjoint finite subsets of $A$ such that for every neighbourhood $U$ of $x$, $\left\{i \in \omega: F_{i} \cap U=\emptyset\right\}$ is finite. For convenience, we denote this relation of $x$ and $\zeta$ by $x(F) \zeta$.

By the definitions, every weakly Fréchet-Urysohn space is a $K$-space. We will show that a regular $K$-space $X$ is a weakly Fréchet-Urysohn space if and only if $X$ has countable tightness.

In this paper, a topological group $G$ always means a Tychonoff space. Also, $\omega, \omega_{1}$ and $c$ denote the first infinite cardinality, the first uncountable cardinality and the cardinality of the continuum, respectively. Further, $\omega(X)$ and $c(X)$ denote the weight and the cellularity of the space $X$, respectively. For other terms and symbols we refer to [2] or [4].

\section{The answers to two questions of homeomorphisms of topological groups}

As a generalisation of the Lindelöf property in topological groups, the $\omega$-narrow property has many interesting results (see [2]). Typical examples of $\omega$-narrow topological groups include the product topological groups $\mathbb{R}^{K}$ which is connected and $\mathbb{Z}^{\kappa}$ which is not connected, where $\mathbb{R}$ and $\mathbb{Z}$ denote the additive groups of reals and integers with the usual topology, respectively. In [2], some open problems about $\omega$ narrow topological groups were left. The following is one of them. 
Problem 2.1. Is every connected topological group homeomorphic to an $\omega$-narrow topological group?

To answer this question, we first recall an interesting topological group constructed by Hartman and Mycielski in [6] from a given topological group $G$.

Let $(G, \cdot)$ be a topological group with identity $e$. Hartman and Mycielski constructed a new topological group as follows. Take $\dot{G}$ to be the set of all functions $f$ defined on the interval $J=[0,1)$ with values in $G$ such that, for some sequence $0=a_{0}<a_{1} \cdots<$ $a_{n}=1, f$ is constant on $\left[a_{k}, a_{k+1}\right)$ for each $k=0, \ldots, n-1$. A binary operation $*$ is defined on $\dot{G}$ such that $(f * g)(x)=f(x) \cdot g(x)$, for all $f, g \in \dot{G}$ and $x \in J$. Then every element $f \in \dot{G}$ has a unique inverse $f^{-1} \in \dot{G}$ defined by $\left(f^{-1}\right)(r)=f(r)^{-1}$ for each $r \in J$. It is easy to see that $(\dot{G}, *)$ is a group with identity $\dot{e}$, where $\dot{e}(r)=e$ for each $r \in J$. For any open neighbourhood $V$ of $e$ in $G$ and a real number $\varepsilon>0$, we define a subset $O(V, \varepsilon)$ of $\dot{G}$ by $O(V, \varepsilon)=\{f \in \dot{G}: \mu(\{r \in J: f(r) \notin V\})<\varepsilon\}$, where $\mu$ is the usual Lebesgue measure on $J$. Let $\mathcal{N}(e)$ be a base for $G$ at $e$ and $\mathcal{N}(\dot{e})=\{O(V, \varepsilon) \mid$ $V \in \mathcal{N}(e), \varepsilon>0\}$. Then $(\dot{G}, *)$ becomes a Hausdorff topological group with $\mathcal{N}(\dot{e})$ being a local base at the identity of $\dot{G}$.

The following important result is due to Hartman and Mycielski [6].

Theorem 2.2. Let $(G, \cdot)$ be a topological group. Then the topological group $(\dot{G}, *)$ is pathwise connected and $G$ is topologically isomorphic to a closed subgroup of $\dot{G}$. If $G$ is metrisable, then $\dot{G}$ is metrisable.

Now we use the topological group $(\dot{G}, *)$ constructed above to give a negative answer to Problem 2.1 [2, Open Problem 3.4.6].

THEOREM 2.3. Let $G$ be the additive group $(\mathbb{R},+)$ of all real numbers with the discrete topology. Then the topological group $\dot{G}$ is not homeomorphic to any $\omega$-narrow topological group.

Proof. Obviously, $G$ is a metrisable topological group. By Theorem 2.2, $\dot{G}$ is a connected metrisable topological group and $\omega(\dot{G}) \geq \omega(G)=c$.

According to [2, Proposition 3.4.5], a first countable $\omega$-narrow topological group has a countable base. Thus, if $\dot{G}$ is homeomorphic to some $\omega$-narrow topological group $H$, then $\omega(H)=\omega$ since $\dot{G}$ is first countable. It follows that $\omega(\dot{G})=\omega$, which contradicts $\omega(\dot{G}) \geq c$.

Recall the definition of balanced groups [2, p. 69]. Assume that $G$ is a topological group. A subset $A$ of $G$ is said to be invariant if $x A x^{-1}=A$ for each $x \in G$. A topological group $G$ is called balanced if it has a local base at the neutral element consisting of invariant subsets.

THeORem 2.4. Suppose that $G$ is a balanced topological group such that for each open neighbourhood $U$ of the neutral element e, there exists a countable subset $M$ of $G$ satisfying $U M U=G$. Then $G$ is an $\omega$-narrow topological group. 
Proof. Take an arbitrary open neighbourhood $V$ of $e$. Since $G$ is a balanced topological group, we can choose a neighbourhood $W$ of $e$ such that $W^{2} \subset V$ and $x W x^{-1}=W$ for each $x \in G$. According to the assumption, there exists a countable subset $M$ of $G$ satisfying $W M W=G$. Since $x W x^{-1}=W$, that is, $x W=W x$, for each $x \in G$, it follows that $W M=M W$. Hence, $G=W M W=M W^{2} \subset M V$, which means that $G$ is an $\omega$-narrow topological group.

Theorem 2.4 gives a partial answer to [2, Open Problem 5.1.12].

Since every abelian topological group is balanced, the following result is obvious.

Corollary 2.5. Suppose that $G$ is a topological group such that for each open neighbourhood $U$ of the neutral element e, there exists a countable subset $M$ of $G$ satisfying $U M U=G$. If $G$ is an abelian group, then $G$ is an $\omega$-narrow topological group.

We now consider the second problem. We know that, for a topological group $G$ with neutral element $e$, its subspace $G \backslash\{e\}$ can fail to be homogeneous. A typical example is the product topological group $\mathbb{R} \times \mathbb{Z}^{\omega}$ of the topological group $\mathbb{R}$ of reals and the topological group $\mathbb{Z}^{\omega}$. But if $G$ is a zero-dimensional topological group with the identity $e$, then the space $G \backslash\{e\}$ is homogeneous (see [2, p. 36]).

Taking account of the homogeneity for every topological group, Arhangel'skii and Tkachenko posed the following question [2, Open Problem 1.4.1].

Problem 2.6. Let $G$ be a zero-dimensional topological group with neutral element $e$. Must the space $X=G \backslash\{e\}$ be homeomorphic to a topological group?

We give a negative answer to this question as follows.

THeORem 2.7. There exists a zero-dimensional topological group $G$ with neutral element e such that the subspace $X=G \backslash\{e\}$ is not homeomorphic to any topological group.

Proof. Let $G$ be the product topological group $D^{\omega_{1}}$, where $D$ is the two-element topological group $\{0,1\}$. Obviously, $G$ is zero-dimensional. We claim that the subspace $X=G \backslash\{e\}$ is not homeomorphic to any topological group.

Assume the contrary, that is, there exists a topological group $H$ which is homeomorphic to $X$. Since $X$ is open in the compact topological group $G$, it is locally compact, which implies that $H$ is locally compact. According to [2, Corollary 3.1.4], a locally compact topological group is paracompact, so that $H$ is paracompact, which implies that $X$ is paracompact. By the Hewitt-Marczewski-Pondiczery theorem in [4] we know that $D^{\omega_{1}}$ is separable, that is, $G$ is separable. It follows that $X$ is separable since $X$ is open in $G$. Therefore, the Souslin number of $X$ is countable. Taking into account that $X$ is paracompact and $c(X)=\omega$, we conclude that $X$ is Lindelöf.

For each ordinal $\alpha<\omega_{1}$, put

$$
K_{\alpha}=\left\{\left(x_{\beta}\right) \in G: x_{\beta}=0, \beta \leq \alpha\right\} ;
$$


then $K_{\alpha}$ is closed in $G$ for each $\alpha<\omega_{1}$. Let $F_{\alpha}=K_{\alpha} \cap X$; then $F_{\alpha}$ is closed in $X$ for each $\alpha<\omega_{1}$. Therefore, we have a family $\left\{F_{\alpha}: \alpha<\omega_{1}\right\}$ consisting of decreasing nonempty closed subsets of $X$. Since $\bigcap_{\alpha<\omega_{1}} K_{\alpha}=\{e\}$ and $e \notin X$, we know that $\bigcap_{\alpha<\omega_{1}} F_{\alpha}=\emptyset$, which implies that the family $\left\{X \backslash F_{\alpha}: \alpha<\omega_{1}\right\}$ is an open cover of $X$. Since $X$ is Lindelöf, $\left\{X \backslash F_{\alpha}: \alpha<\omega_{1}\right\}$ has a countable subcover $\left\{X \backslash F_{\alpha_{i}}: i \in \omega\right\}$, that is, $\bigcap_{i \in \omega} F_{\alpha_{i}}=\emptyset$. Taking into account that $\omega_{1}$ is a regular cardinality, we can find an ordinal number $\gamma$ such that $\gamma<\omega_{1}$ and $\gamma>\alpha_{i}$ for each $i \in \omega$. Since $\left\{F_{\alpha}: \alpha<\omega_{1}\right\}$ is a decreasing family, we conclude that $F_{\gamma} \subset \bigcap_{i \in \omega} F_{\alpha_{i}}=\emptyset$, which is contradiction.

\section{Some results on $K$-topological groups}

If a topological group $G$ is a $K$-space, then we will call it a $K$-topological group.

In [9], Malykhin and Tironi investigated weakly Fréchet-Urysohn spaces and Pytkeev spaces. The following open problem was posed in [9, Question 6.4].

Problem 3.1. Must a compact $K$-space $X$ have countable tightness?

We now consider this problem for compact topological groups; equivalently, we ask whether a compact $K$-topological group must have countable tightness. To answer this question, we first recall a theorem in [2, Theorem 4.2.1].

THEOREM 3.2. If $G$ is a nonmetrisable compact topological group of weight $\tau$, then the space $D^{\tau}$ is homeomorphic to a subspace of $G$, where $D$ is the two-element topological group $\{0,1\}$.

Theorem 3.2 is an easy corollary from a famous theorem (every compact topological group $G$ is a dyadic compactum) in [7] and a general result of Engelking on dyadic compacta in [3]. From Theorem 3.2 we can obtain the important theorem in [1]: every compact topological group with countable tightness is metrisable.

We now show that for compact topological groups, Problem 3.1 has an affirmative answer.

Theorem 3.3. Suppose that $G$ is a compact topological group. If $G$ is a K-topological group, then $G$ is metrisable.

Proof. Assume the contrary, that is, that $G$ is not a metrisable topological group. Then there exists a cardinal number $\tau$ such that $\omega(X)=\tau$ and $\tau \geq \omega_{1}$. According to Theorem 3.2, the space $D^{\tau}$ is homeomorphic to a subspace of $G$. Since $D^{\omega_{1}}$ is homeomorphic to a subspace of $D^{\tau}$, it follows that $D^{\omega_{1}}$ is homeomorphic to a subspace of $G$.

Let $Y$ be the subspace of $D^{\omega_{1}}$ consisting of all elements of $\left(x_{\alpha}\right)$ such that, for some successor ordinal $\beta<\omega_{1}$, the $\alpha$ th coordinate $x_{\alpha}$ is 0 whenever $\alpha<\beta$, and all other coordinates of $\left(x_{\alpha}\right)$ are 1 . Obviously, the cardinality of $Y$ is $\omega_{1}$. We claim that $Y$ is a discrete subspace of $D^{\omega_{1}}$. Indeed, for an arbitrary element $y=\left(y_{\alpha}\right)$ of $Y$, suppose that $\gamma$ is the first coordinate of $y$ which equals 1 . Then, by the choice of $Y, \gamma$ is a successor 
ordinal. We denote the predecessor of $\gamma$ by $\gamma-1$. Put

$$
U=\left\{\left(x_{\alpha}\right) \in D^{\omega_{1}}: x_{\gamma}=1, x_{\gamma-1}=0\right\}
$$

then the subset $U$ is an open neighbourhood of $y$ in $D^{\omega_{1}}$ and $U \cap Y=\{y\}$. Therefore, $Y$ is a discrete subspace of $D^{\omega_{1}}$.

It is easy to see that the element $x=\left(0_{\alpha}\right)$ of $D^{\omega_{1}}$ satisfies $x \in \bar{Y} \backslash Y$ where $0_{\alpha}=0$ for each ordinal $\alpha<\omega_{1}$. Since $G$ is a $K$-space, then it follows from the hereditariness of $K$-spaces that $D^{\omega_{1}}$ is a $K$-space. Thus, there exists a sequence $\zeta=\left\{K_{i}: i \in \omega\right\}$ of disjoint compact subsets of $Y$ satisfying $x(K) \zeta$. Since each $K_{i}$ is compact and $Y$ is discrete, it follows that each $K_{i}$ is a finite subset. Therefore, $\bigcup_{i \in \omega} K_{i}$ is a countable subset of $D^{\omega_{1}} \backslash\{x\}$. It follows from the choice of $Y$ that there exists a $\beta<\omega_{1}$ such that the $\beta$ th coordinate of each element of $\bigcup_{i \in \omega} K_{i}$ is 1 . Put $V=\left\{\left(t_{\alpha}\right) \in D^{\omega_{1}}: t_{\beta}=0\right\}$; then $V$ is an open neighbourhood of $x$. However, $V \cap\left(\bigcup_{i \in \omega} K_{i}\right)=\emptyset$, which contradicts $x(K) \zeta$.

Hence, $D^{\omega_{1}}$ is not a $K$-space. Since $K$-spaces are hereditary, we know that $G$ is not a $K$-space, which is a contradiction. Thus, $G$ is metrisable.

From the proof of Theorem 3.3 we can see the topological group $D^{\omega_{1}}$ is not a $K$-space. Thus the following result is obvious.

Corollary 3.4. A compact topological group need not be a $K$-space. In particular, an $\omega$-narrow topological group need not be a $K$-space.

In Theorem 3.3 the compactness of $G$ cannot be replaced by countable compactness. A suitable example is the $\Sigma$-product of $\omega_{1}$ copies of a two-element topological group $D$, which is countably compact. We denote this group by $H$. Then $H$ is a FréchetUrysohn space, which implies that $H$ is a $K$-space. However, $H$ is nonmetrisable. Another example is the $\sigma$-product of $\omega_{1}$ copies of $D$, which is a $\sigma$-compact space. We denote this group by $M$. Since $M$ is a subspace of $H$, it is a $K$-space. Taking account of the fact that $M$ is dense in $H$, we have $\chi(M)=\chi(H)$, so that $M$ is nonmetrisable either. Therefore the compactness of $G$ in Theorem 3.3 cannot be replaced by $\sigma$-compactness.

It turns out that Theorem 3.3 remains valid if one replaces the compactness of $G$ by Čech-completeness. To prove this, we first need an auxiliary result.

Recall that a topological group $G$ is feathered [2, p. 235] if it contains a nonempty compact subset $K$ of countable character in $G$, that is, $K$ has a countable neighbourhood base in $G$.

Theorem 3.5. A feathered topological group $G$ is metrisable if and only if it is a K-topological group.

Proof. Necessity is obvious. It remains to verify sufficiency.

Since $G$ is feathered, according to [2, Lemma 4.3.19], there exists a compact subgroup $H$ of $G$ such that the left coset space $G / H$ is metrisable. By the condition that $G$ is a $K$-topological group, we know that $H$ is a $K$-topological group. Then, by Theorem 3.3, $H$ is metrisable. Since $H$ and $G / H$ are both first countable, 
by [2, Corollary 1.5.21], we know that $G$ is first countable. Therefore, $G$ is metrisable.

Since every Čech-complete topological group is feathered, the following result is obvious.

COROLlary 3.6. Every Čech-complete K-topological group is metrisable. In particular, each locally compact $K$-topological group is metrisable.

Since every sequential space is a $K$-space, we have the following result.

Corollary 3.7. Every Čech-complete sequential topological group is metrisable. In particular, each locally compact sequential topological group is metrisable.

Now we consider $K$-topological groups with countable pseudocharacter. We will show the following result.

THEOREM 3.8. Every K-topological group with countable pseudocharacter has countable tightness.

The proof of this theorem will follow from the next two results. First, we recall the definition of $G_{\delta}$-diagonal. A topological space $X$ is said to have a $G_{\delta}$-diagonal if there exists a sequence $\left\{\mathcal{V}_{i}: i \in \omega\right\}$ of open covers of $X$ such that $\bigcap_{i \in \omega} \operatorname{st}\left(x, \mathcal{V}_{i}\right)=\{x\}$ for every $x \in X$, where $\operatorname{st}\left(x, \mathcal{V}_{i}\right)=\bigcup\left\{V \in \mathcal{V}_{i}: x \in V\right\}$.

Lemma 3.9. A regular $K$-space $X$ with a $G_{\delta}$-diagonal has countable tightness.

Proof. Suppose that $x \in X, A \subset X$ and $x \in \bar{A} \backslash A$. Since $X$ is a $K$-space, there exists a sequence $\zeta=\left\{K_{i}: i \in \omega\right\}$ consisting of disjoint compact subsets of $A$ such that $x(K) \zeta$. In particular, $x \in \overline{\bigcup_{i \in \omega} K_{i}}$. Since $X$ has a $G_{\delta}$-diagonal, for each $i \in \omega$, the subspace $K_{i}$ also has a $G_{\delta}$-diagonal. Then, for each $i \in \omega, K_{i}$ is a separable and metrisable, which follows from the fact that $K_{i}$ is compact and has a $G_{\delta}$-diagonal. Choose a countable dense subset $C_{i}$ of $K_{i}$ for every $i \in \omega$. Then $B=\bigcup_{i \in \omega} C_{i}$ is a countable subset of $A$ and $x \in \bar{B}$. Hence, $X$ has countable tightness.

Since every submetrisable space has a $G_{\delta}$-diagonal, the following result is obvious.

Corollary 3.10. Every regular submetrisable K-space has countable tightness.

Lemma 3.11. Every topological group $G$ with countable pseudocharacter has a $G_{\delta^{-}}$ diagonal.

Proof. Since $G$ has countable pseudocharacter, there exists a sequence $\left\{U_{i}: i \in \omega\right\}$ of open subsets of $G$ such that $\bigcap_{i \in \omega} U_{i}=\{e\}$, where $e$ is the identity of $G$. Taking account of the fact that $G$ is a topological group, we can find another sequence $\left\{V_{i}: i \in \omega\right\}$ of open symmetric neighbourhoods of $e$ such that $V_{i+1}^{2} \subset V_{i} \cap U_{i}$ for each $i \in \omega$. By virtue of $\left\{V_{i}: i \in \omega\right\}$ we have a sequence $\left\{\mathcal{V}_{i}: i \in \omega\right\}$ of open covers of $G$ such that $\mathcal{V}_{i}=\left\{x V_{i}: x \in G\right\}$ for each $i \in \omega$. 
We claim that $\bigcap_{i \in \omega} s t\left(x, \mathcal{V}_{i}\right)=\{x\}$ for every $x \in G$. Assuming the contrary, there exist two distinct points $y$ and $z$ such that $z \in \bigcap_{i \in \omega} \operatorname{st}\left(y, \mathcal{V}_{i}\right)$. Then, for each $i \in \omega$, there exists a point $x_{i} \in G$ such that $\{y, z\} \subset x_{i} V_{i}$, that is, we can find two points $u_{i}, v_{i}$ in $V_{i}$ such that $y=x_{i} u_{i}$ and $z=x_{i} v_{i}$. It follows that $x_{i}=y u_{i}^{-1}$, which implies that

$$
z=y u_{i}^{-1} v_{i} \in y V_{i}^{-1} V_{i}=y V_{i}^{2} \subset y U_{i} \quad \text { for every } i \in \omega .
$$

Since $\bigcap_{i \in \omega} U_{i}=\{e\}$ and $G$ is homogeneous, $\bigcap_{i \in \omega} y U_{i}=\{y\}$ which implies $y=z$. This is a contradiction. Hence, $G$ has a $G_{\delta}$-diagonal.

Lemma 3.11 fails to be valid in the category of topological spaces, even for compact spaces. A suitable example is the Alexandroff double circle [4, Example 3.1.26] $X$ which is compact and first countable. However, the Souslin number of $X$ is $c$, which means that $X$ does not have a $G_{\delta}$-diagonal. Otherwise, $X$ would be separable and metrisable, which implies that the Souslin number of $X$ is countable. This is a contradiction.

Proof of Theorem 3.8. The theorem follows directly from Lemmas 3.9 and 3.11.

We recall an interesting result given by Arhangel'skii and Tkachenko [2, Lemma 3.3.22].

Lemma 3.12. The following conditions are equivalent for a topological group G:

(a) every compact subspace of $G$ is first countable;

(b) every compact subspace of $G$ is metrisable.

THeOREM 3.13. Assume that $G$ is a K-topological group. If every compact subspace of $G$ is first countable, then $G$ has countable tightness.

Proof. Suppose that $x \in G, A \subset G$ and $x \in \bar{A} \backslash A$. Since $G$ is a $K$-space, there exists a sequence $\zeta=\left\{K_{i}: i \in \omega\right\}$ of disjoint compact subsets of $A$ such that $x(K) \zeta$. By virtue of the assumption, we know that $K_{i}$ is first countable for every $i \in \omega$. According to Lemma 3.12, each $K_{i}$ is metrisable, so that the compact subspace $K_{i}$ is separable. Therefore, the subspace $\bigcup_{i \in \omega} K_{i}$ is separable. From $x \in \overline{\bigcup_{i \in \omega} K_{i}}$ we can conclude that $G$ has countable tightness.

We know that every weakly Fréchet-Urysohn space is a $K$-space but the converse is not true. So it is interesting to ask under what conditions a $K$-space is a weakly Fréchet-Urysohn space. The following result gives a complete answer to this question.

THeorem 3.14. A regular $K$-space $X$ is a weakly Fréchet-Urysohn space if and only if $X$ has countable tightness.

Proof. We begin with necessity. By the definition of weakly Fréchet-Urysohn spaces, every weakly Fréchet-Urysohn space has countable tightness.

We now prove sufficiency. Assume $x \in X$ and $A \subset X$ satisfying $x \in \bar{A} \backslash A$. Since $X$ has countable tightness, there exists a countable subset $B$ of $A$ satisfying $x \in \bar{B} \backslash B$. It follows from the fact $X$ is a $K$-space that there exists a sequence $\zeta=\left\{K_{i}: i \in \omega\right\}$ 
consisting of disjoint compact subsets of $B$ satisfying $x(K) \zeta$. Since each $K_{i}$ is compact and countable, according to [4, Theorem 3.1.19 ], it is separable and metrisable. By [8, Lemma 13.2], each $K_{i}$, as a countable compact metrisable space, has isolated points, where by an isolated point we mean it is isolated in the subspace $K_{i}$. In addition, each accumulation point (if it exists) on $K_{i}$ is a limit point of some countable isolated points on $K_{i}$ for every $i \in \omega$. If there exists an infinite subfamily $\eta$ of $\zeta$ such that each element of $\eta$ contains only isolated points, then there is nothing to prove, since a compact subset consisting of isolated points is a finite subset.

Now we assume that for each $i \in \omega, K_{i}$ has accumulation points. Let $H_{i}=\left\{x_{i, m}\right.$ : $m \in \omega\}$ be the subset of accumulation points on $K_{i}$. For each $i, m \in \omega$, take a sequence $\left\{x_{i, m}^{k}: k \in \omega\right\} \subset K_{i} \backslash H_{i}$ such that $\left\{x_{i, m}^{k}: k \in \omega\right\}$ converges to $x_{i, m}$. Put $F_{i, m}^{l}=\left\{x_{i, m}^{k}: k \geq l\right\}$, $i, m, l \in \omega$. We consider the following two cases.

Case 1. For each neighbourhood $U$ of $x,\left\{i \in \omega: U \cap H_{i}=\emptyset\right\}$ is finite. Therefore, $\xi=\left\{F_{i, m}^{l}: i, m, l \in \omega\right\}$ is a countable $\pi$-network of $X$ at $x$ consisting of infinite subsets. According to [9, Proposition 1.1], there exists a countably infinite sequence $\lambda$ of finite subsets of $B$ satisfying $x(F) \lambda$.

Case 2. There exists a neighbourhood $U$ of $x$ such that $\left\{i \in \omega: U \cap H_{i}=\emptyset\right\}$ is infinite. Suppose that $M=\left\{i \in \omega: U \cap H_{i}=\emptyset\right\}$. According to the definition of $\zeta$, we have the following conclusion: for each neighbourhood $V$ of $x$, $\left\{i \in M: V \cap\left(K_{i} \backslash H_{i}\right)=\emptyset\right\}=$ $\left\{i \in M: V \cap K_{i}=\emptyset\right\}$ is finite (*). Choose a neighbourhood $O$ of $x$ satisfying $\bar{O} \subset U$, then $O \cap K_{i}=O \cap\left(K_{i} \backslash H_{i}\right)$ for every $i \in M$. According to $(*)$, we can assume that $O \cap\left(K_{i} \backslash H_{i}\right)$ is not empty for each $i \in M$. Thus, each $\bar{O} \cap K_{i}=\bar{O} \cap\left(K_{i} \backslash H_{i}\right)$ is compact and discrete. It follows that $T_{i}=O \cap\left(K_{i} \backslash H_{i}\right)$ is finite for each $i \in M$. For any two distinct $m, n \in M, T_{m} \cap T_{n} \subset K_{m} \cap K_{n}=\emptyset$. For each neighbourhood $W$ of $x$, it follows from $(*)$ that

$$
\left\{i \in M: W \cap T_{i}=\emptyset\right\}=\left\{i \in M:(W \cap O) \cap\left(K_{i} \backslash H_{i}\right)=\emptyset\right\}
$$

is finite. Therefore, $x(F)\left\{T_{i}: i \in M\right\}$.

Lemma 3.15. Suppose that $X$ is a $T_{2} K$-space, $x \in X, A \subset X$ and $x \in \bar{A} \backslash A$. Then there exist two disjoint subsets $B, C$ of $A$ satisfying $x \in \bar{B}$ and $x \in \bar{C}$.

Proof. By virtue of the assumption, there exists a sequence $\zeta=\left\{K_{i}: i \in \omega\right\}$ of disjoint compact subsets of $A$ satisfying $x(K) \zeta$. Put

$$
B=\bigcup_{i \in \omega} K_{2 i} \text { and } C=\bigcup_{i \in \omega} K_{2 i+1} ;
$$

then $B \cap C=\emptyset, x \in \bar{B}$ and $x \in \bar{C}$, which follow from $x(K) \zeta$.

The following result gives an example of a countable topological group which is not a $K$-space, so the countable tightness and countable pseudocharacter cannot make a topological group be a $K$-space. 
Corollary 3.16. Suppose that $\beta \omega$ is the Stone-Čech compactification of $\omega, p \in$ $\beta \omega \backslash \omega$. Let $X=\omega \cup\{p\}$ be the subspace of $\beta \omega$. Then the countable free topological group $F(X)$ is not a $K$-space.

Proof. Obviously, $p \in \bar{\omega} \backslash \omega$. Since $p$ is a free ultrafilter, there do not exist two disjoint subsets $B, C$ of $\omega$ such that $x \in \bar{B}$ and $x \in \bar{C}$. By Lemma 3.15, $X$ is not a $K$-space. Since $X$ is a subspace of the free topological group $F(X), F(X)$ is not a $K$-space.

\title{
References
}

[1] A. V. Arhangel'skii and V. I. Ponomarev, 'On dyadic bicompacta', Soviet. Math. Dokl. 9 (1968), $1220-1224$.

[2] A. V. Arhangel'skii and M. Tkachenko, Topological Groups and Related Structures (Atlantis Press, Amsterdam, 2008).

[3] R. Engelking, 'Cartesian products and dyadic spaces', Fund. Math. 57 (1965), 287-304.

[4] R. Engelking, General Topology (Heldermann, Berlin, 1989).

[5] I. I. Guran, 'On topological groups close to being Lindelöf', Soviet Math. Dokl. 23 (1981), 173-175.

[6] S. Hartman and J. Mycielski, 'On embeddings of topological groups into connected topological groups', Colloq. Math. 5 (1958), 167-169.

[7] L. N. Ivacovskij, 'On a hypothesis of P. S. Alexandroff', Dokl. Akad. Nauk SSSR 123 (1958), 785-786.

[8] Y. Kodama and K. Nagami, General Topology (Iwanami Shoten, Tokyo, 1974).

[9] V. I. Malykhin and G. Tironi, 'Weakly Fréchet-Urysohn and Pytkeev spaces', Topology Appl. 104 (2000), 181-190.

\author{
HANFENG WANG, Institute of Mathematics, Nanjing Normal University, \\ Nanjing 210046, PR China \\ and \\ Department of Mathematics, Shandong Agricultural University, \\ Taian 271018, PR China \\ e-mail: whfeng@sdau.edu.cn
}

WEI HE, Institute of Mathematics, Nanjing Normal University,

Nanjing 210046, PR China

e-mail: weihe@njnu.edu.cn 